\title{
Parking Lot Optimal Routing Based on Grey Entropy Relation Grade Multi-Attribute Decision Making
}

\author{
Zhang Yuru, Zhao Wuchen, Li Hui, Su Xiaodong and Zhao Ming \\ School of Computer and Information Engineering \\ Harbin University of Commerce \\ hsdzyr@163.com
}

\begin{abstract}
By analyzing the drivers' main consideration about how to choose a parking space, this paper is committed to path optimization of parking lot by grey entropy relation grade multi-attribute decision making. The decision attribute matrix is identified by driving distance which is deduced through Dijkstra algorithm, walking distance deduced through Euclidean distance and parking space environment value deduced through the expectation of triangular fuzzy number. Finally, an example would be analyzed to verify the feasibility of the method.
\end{abstract}

Keywords : parking lot optimal routing; grey entropy relation grade; Multiple Attribute Decision Making (MADM)

\section{Introduction}

The establishment of the internal parking guidance system is probable along with the development of techniques such as communication, dynamic traffic signs, parking space detection. In recent years, parking guidance system has been gradually introduced by many car parking management company, and newly built large parking lots of this system have been set up. But in the process of system providing parking guidance to driver, it's easy to have a dissatisfied about the system's assigning parking space, then the parking guidance system is not effect. The root cause is that the existing parking guidance system is not considering the perspective of the driver and just directly give a random parking space or a shortest path method based parking space [1]. Therefore, how to find drivers satisfying parking space and make drivers trust parking guidance system will be resolved in turn to achieve the parking lots operational efficency.

This paper calculates the driving distance, walking distance and the environment value of parking lots as the decision attribute value by analyzing the main influence factors of parking choices, then makes the optimal routing take advantage of grey entropy relation grade MADM.

\section{Parking Process Analysis}

\subsection{Parking under the Condition without Induction}

Parking process refers to a series of search decisions process about a driver driving a car from the entrance of parking lot to parking space, it's a kind of micro behavior. On the premise without induction, the car parking process is as shown in Fig.1. Drivers are looking for effective parking space to spare when driving into a parking lot. The surrounding conditions about the parking space and the situations of parking space itself is considered by a driver when he is finding an effective parking space. If the driver satisfies and accepts this parking space, he parks and left. If not, he will continue to looking for a next effective parking space, until the desired one. The number of parking 
spaces is so large that the driver is able to find a parking space quickly. Although that, he maybe cost lots of time to choose because the parking spaces is so much. At the peak time, it is hard to find a parking space in view of the closed environment of the parking lot.

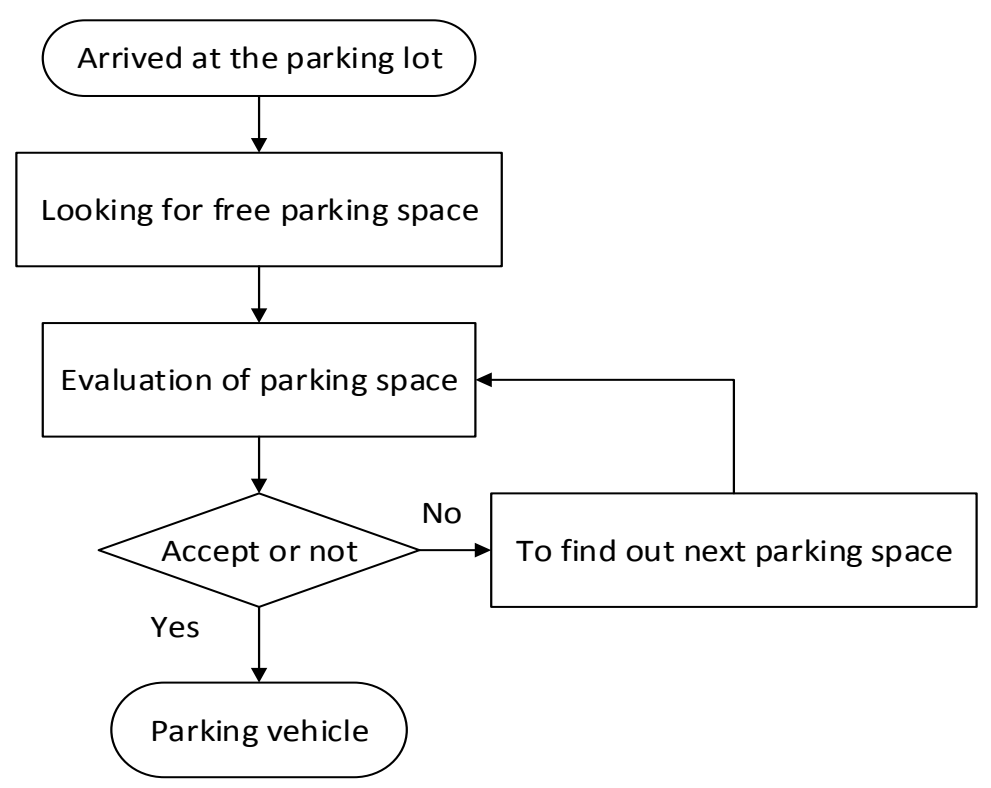

Figure 1. Parking Process under the Condition without Induction

\subsection{Parking under the Condition with Induction}

If the parking lot has parking space navigation system, the parking process is shown in Fig.2. The parking space navigation system startups to provide an optimal route for driver when he was diving into the parking lot.

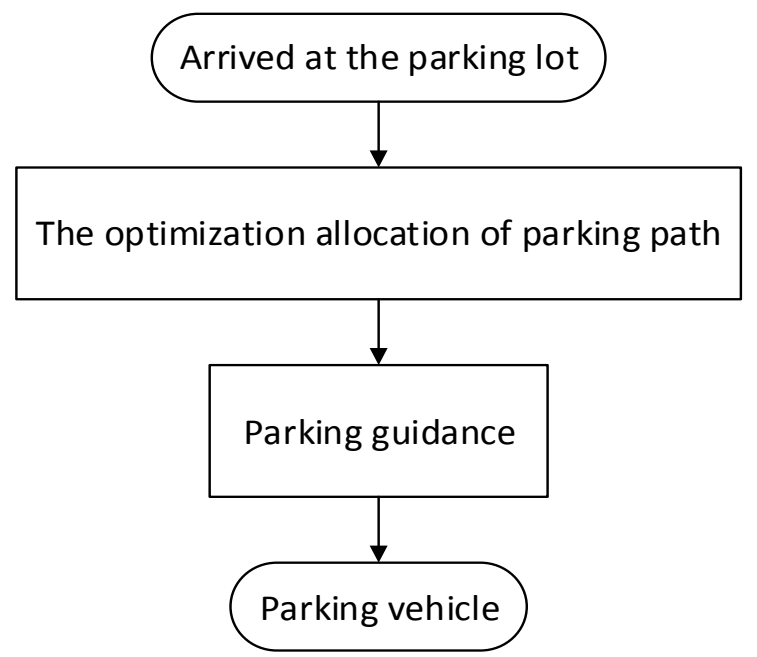

\section{Figure 2. Parking Process under the Condition with Induction}

\subsection{Comparison of Two Parking Processes}

By comparing two processes above, the second one is greatly simplifier as system helping driver to choose parking space. Even so, it's just happened with the accept 
parking guidance, if not, the parking guidance system will be useless as driver will continue to look for the next one. Therefore, the design of the system should contain the factors where drivers choose a parking space.

\section{Main Influence Factors on Choosing Parking Space}

The factors when drivers choose parking space are need to analysis before optimal routing. The main factors are driving distance, walking distance, safety and the both sides of selected parking space's take up situations, for the outdoor parking lots, the length of shade is also considered [2].

\subsection{Driving Distance}

Driving distance refers to the shortest distance between parking lot's entrance and the parking space, and we use the Dijkstra algorithm to determine the driving distance. Edsgar Wybe Dijkstra, a Dutch computer scientist, put forward Dijkstra algorithm in 1959, it computes the shortest path from a vertex to other vertex [3]. The main idea of this algorithm is as follows:

(1) The vertex set $V$ of the graph is divided into two vertex sets $S$ and $T, S$ is confirmed vertex set and just have source point at the start, $T=V-S$ is unconfirmed vertex set;

(2) The point of vertex set $T$ is transferred through the order of increasing way to the vertex set $S$, it comply with the following rules: the distance of the source point $V_{0}$ to other points in vertex set $S$ is less than or equal to the distance of the source point $V_{0}$ to all points in vertex set $T$;

(3) In the end, the vertex set $S$ contained the length of the source point to the target point, the vertex set $T$ contained the length of the source point to the point before the target point.

\subsection{Walking Distance}

Walking distance refers to the distance parking space to the exit of parking lot or internal elevator, and we use the Euclidean distance to determine the walking distance. Euclidean distance is the length of the straight range between two points, the walking distance of the driver left the parking lot is a fuzzy value due to people's subjective initiative, because people usually walk as short as possible, it is feasible for measuring walking distance with Euclidean distance.

\subsection{Parking Space Environment Value}

The environmental information of parking space is the occupancy situations of both sides and the shape itself. Fig.3 illustrates the occupancy situations of both sides about a parking space. (a) exhibites the both sides of the parking space has been occupied, (b) demonstrates the one side of the parking space has been occupied and (c) shows no sides of parking space has been occupied.

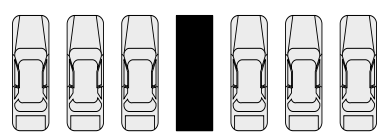

(a) Both sides occupation

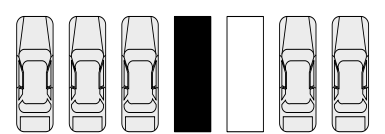

(b) One side occupation

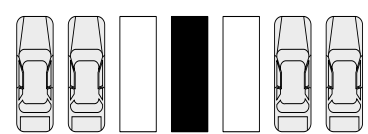

(c) No sides occupation

Figure 3. Occupancy Situations of Parking Space's both Sides 
Fig.4 illustrates the shape of a parking space. (a) exhibites the lined shape parking space, (b) demonstrates the $\mathrm{T}$ shape parking space and (c) shows the bias shape parking space.
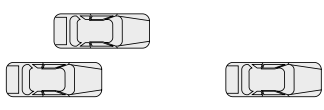

(a) Lined shape

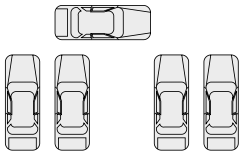

(b) T shape

(c) Bias shape

Figure 4. The Shape of a Parking Space

Calculation expectation of triangular fuzzy number is shown to determine the parking environment information value. Triangular fuzzy number put forward by Zadeh in 1965, it's solved the problem of uncertain environment [4]. A fuzzy set for domain $U$ makes $\forall x \in U$ have matching with membership $\mu(x) \in[0,1], \mu$ is known as membership function.

$$
\mu(x)=\left\{\begin{array}{c}
\frac{x}{m-s}-\frac{s}{m-s}, x \in[s, m] \\
\frac{x-u}{m-u}-\frac{u}{m-u}, x \in[m, u] \\
0, \text { others }
\end{array}\right.
$$

$s$ and $u$ are lower limit and upper limit of confidence for triangular fuzzy number, $m$ is the biggest possible value, and a triangular fuzzy number is represented by $(s m u)$.

Assuming a triangular fuzzy number $a=\left(a_{I}, a_{m}, a_{u}\right), 0<a_{\mathbb{I}} \leq a_{m} \leq a_{u}$, its expectation is defined as follows [5].

$$
E(a)=\frac{(1-\lambda) a_{I}+a_{m}+\lambda a_{u}}{2}
$$

Usually, $\lambda=0.5$.

According rules: no sides occupation is the best, and both sides occupation is the worst, else lined shape parking space is hard to park and bias shape parking space is easy to park, it makes order in parking difficulty and give value 0.1-0.9 to them. The confidence limit is \pm 0.05 ,then compute matching expectations.

\section{Optimal Routing Choosing}

\subsection{Relevant Concepts}

\subsubsection{Fundamental Theory of Entropy}

The concept of entropy is put forward by a German physicist and mathematician, Rudolf Julius Emanuel Clausius, using to represent the degree of chaos about energy in a system. The entropy is maximum when a system's energy is completely obey uniform 
distribution [6]. In 1948, Claude Elwood Shannon, American mathematician and the founder of information theory, introduced the concept of entropy to information theory and puts forward the concept of information entropy [7].

(1) Information entropy

Hypothesis $X=\left\{x_{i} \mid i=1,2, \cdots, n\right\}, \forall i, x_{i} \geq 0$ is a discrete random variable, its domain of definition is $R=\left\{x_{1}, x_{2}, x_{3}, \cdots\right\}$. Suppose $p_{i}=P\left(X=x_{i}\right)$, then the information entropy of $X=\left\{x_{i} \mid i=1,2, \cdots, n\right\}, \forall i, x_{i} \geq 0$ is defined as follows.

$$
H(X)=-\sum_{i=1}^{\infty} p_{i} \log p_{i}
$$

(2) Grey entropy and grey entropy increase theory

Suppose a discrete sequence $X=\left\{x_{i} \mid i=1,2, \cdots, n\right\}, \forall i, x_{i} \geq 0$ and $\sum_{i=1}^{n} x_{i}=1$, the grey entropy of $X=\left\{x_{i} \mid i=1,2, \cdots, n\right\}, \forall i, x_{i} \geq 0$ is defined as follows.

$$
H(X)=-\sum_{i=1}^{n} x_{i} \ln x_{i}
$$

It can be seen by the concept of grey entropy and information entropy, grey entropy is obtained from limited information, and information entropy is obtained from unlimited information [8].

Grey entropy increase theory: the grey entropy value of the sequence will increase when any changes make the sequence equality.

(3) Balanced degree

Grey entropy is a constant only related to the number of elements when the sequence is obey uniform distribution, from the grey entropy theorem. The balanced degree of $X=\left\{x_{i} \mid i=1,2, \cdots, n\right\}, \forall i, x_{i} \geq 0$ is defined as follows.

$$
B=\frac{H(X)}{H_{\max }}
$$

As the value is greater, the sequence is more approximate equilibrium[9].

\subsubsection{Fundamental Theory of Grey System}

In many practical problems of the objective world, the system is divided into black, grey and white depending on the structure, characteristics and parameters. The black system is refers to a system of information completely unknown, the white one which is internal mechanism all clear and the grey one is a system between them [10]. In practical problems, there is a lot of gray system, the absolute black or white system is very little.

(1) Data transformation technology

Suppose a sequence $x=(x(1), x(2), \cdots, x(n))$, the data transformation $x$ to $y$ is defined as follows.

$$
\begin{gathered}
f(x(k))=y(k), k=1,2, \cdots, n \\
f: x \rightarrow y
\end{gathered}
$$

Then the interval value transformation is defined as follows.

$$
f(x(k))=\frac{x(k)-\min _{k} x(k)}{\max _{k} x(k)-\min _{k} x(k)}=y(k)
$$

(2) Correlation analysis 
The reference sequence is given as follows.

$$
x_{0}=\left\{x_{0}(k) \mid k=1,2, \cdots, n\right\}=\left(x_{0}(1), x_{0}(2), \cdots, x_{0}(n)\right)
$$

Among them, $k$ is refers to time.

Suppose there having $m$ sequences is given as follows.

$$
x_{i}=\left\{x_{i}(k) \mid k=1,2, \cdots, n\right\}=\left(x_{i}(1), x_{i}(2), \cdots, x_{i}(n)\right), i=1,2, \cdots, m
$$

The correlation coefficient of compare sequence and reference sequence in time is defined as followed.

$$
\xi_{i}(k)=\frac{\min _{s} \min _{t}\left|x_{0}(t)-x_{s}(t)\right|+\rho \max _{s} \max _{t}\left|x_{0}(t)-x_{s}(t)\right|}{\left|x_{0}(t)-x_{i}(t)\right|+\rho \max _{s} \max _{t}\left|x_{0}(t)-x_{s}(t)\right|}
$$

The distinguish coefficient is $\rho \in[0,1]$. Generally speaking, the $\rho$ is greater, the resolution is greater; vice versa. Correlation coefficient is mainly described the connection degree of compare sequence and reference sequence at a certain moment. Every moment has an associated number. Correlation coefficient need to be dealt to prevent too scattered about each correlation number. The correlation degree of $x_{i}$ to $x_{0}$ is defined as follows.

$$
r_{i}=\frac{1}{n} \sum_{k=1}^{n} \xi_{i}(k)
$$

Correlation degree is associated numbers to centralized processing and average each of them.

\subsection{Decision Making Process}

(1) Using driving distance, walking distance and parking space environment information value to determine decision attribute matrix $R^{\prime}$;

$$
R^{s}=\left\{r_{i j}^{s} \mid i=1,2, \cdots, m ; j=1,2, \cdots, n\right\}
$$

Among them, $i=1,2, \cdots, m$ expresses the number of parking space, $j=1,2, \cdots, n$ expresses the number of attribute.

(2) Obtain $\mathrm{R}=\left(r_{\mathrm{ij}}\right)_{(\mathrm{m \times n})}$ (斜体) by dimensionless standardization of decision attribute matrix;

$$
\begin{aligned}
& r_{i j}= \frac{r_{i j}^{\prime}-\min _{i} r_{i j}^{\prime}}{\max _{i} r_{i j}^{\prime}-\min _{i} r_{i j}^{\prime}} \quad i \in \text { Efficiency indicators } \\
& r_{i j}=\frac{\max _{i} r_{i j}^{\prime}-r_{i j}^{\prime}}{\max _{i} r_{i j}^{\prime}-\min _{i} r_{i j}^{\prime}} \quad i \in \text { Cost type indicators }
\end{aligned}
$$

Here, efficiency indicators is the value which is better as bigger, and parking space environment information value belongs to efficiency indicators. Cost type indicators is the value which is better as smaller, and driving distance and walking distance belong to cost type indicators.

(3) Determining information entropy $H_{i}$ of $r_{i j}$; 


$$
H_{i}=-\sum_{j=1}^{n} r_{i j} l n r_{i j}
$$

(4) Determining balanced degree $B_{i}$ of information entropy;

$$
B_{i}=\frac{H_{i}}{H_{\max }}
$$

Among them, $H_{\max }$ is the maximum of all information entropy.

(5) Determining entropy attribute matrix $V$;

$$
V=\left[\begin{array}{cccc}
H_{1} r_{11} & H_{1} r_{12} & \ldots & H_{1} r_{1 n} \\
H_{2} r_{21} & H_{2} r_{22} & & H_{2} r_{2 n} \\
\vdots & \vdots & \ddots & \vdots \\
H_{m} r_{m 1} & H_{m} r_{m 2} & \cdots & H_{m} r_{m n}
\end{array}\right]
$$

(6) Determining ideal solution set $e^{*}$;

$$
e^{*}=\left\{V_{j}^{*} \mid V_{j}^{*}=\max _{i} V_{i j} \text { or } \min _{i} V_{i j}\right\}
$$

Here, the selected one depend on the type of indicators.

(7) Determining the grey correlation degree $R_{o i}$;

$$
R_{\text {oi }}=\frac{1}{m} \sum_{k=1}^{m} \frac{\min _{i} \min _{k}\left|V_{k}^{*}-V_{k}^{i}\right|+\rho \max _{i} \max _{k}\left|V_{k}^{*}-V_{k}^{i}\right|}{\left|V_{k}^{*}-V_{k}^{i}\right|+\rho \max _{i} \max _{k}\left|V_{k}^{8}-V_{k}^{i}\right|}
$$

(8) Determining equilibrium close degree $\omega$;

$$
\omega=B_{i} R_{o i}
$$

(9) Sorting the value of equilibrium close degree. The maximum value mapping the optimal parking location. Since 2.1 has been given the shortest path of this parking space, it's the optimal route.

\section{Example Analysis}

The situations of free parking space in a parking lot at a moment has been shown as Fig.5. 
G

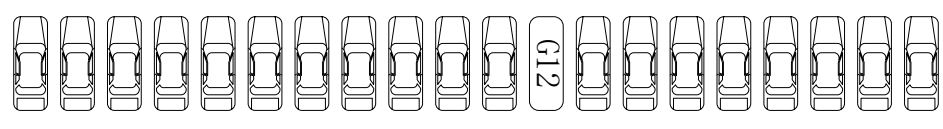
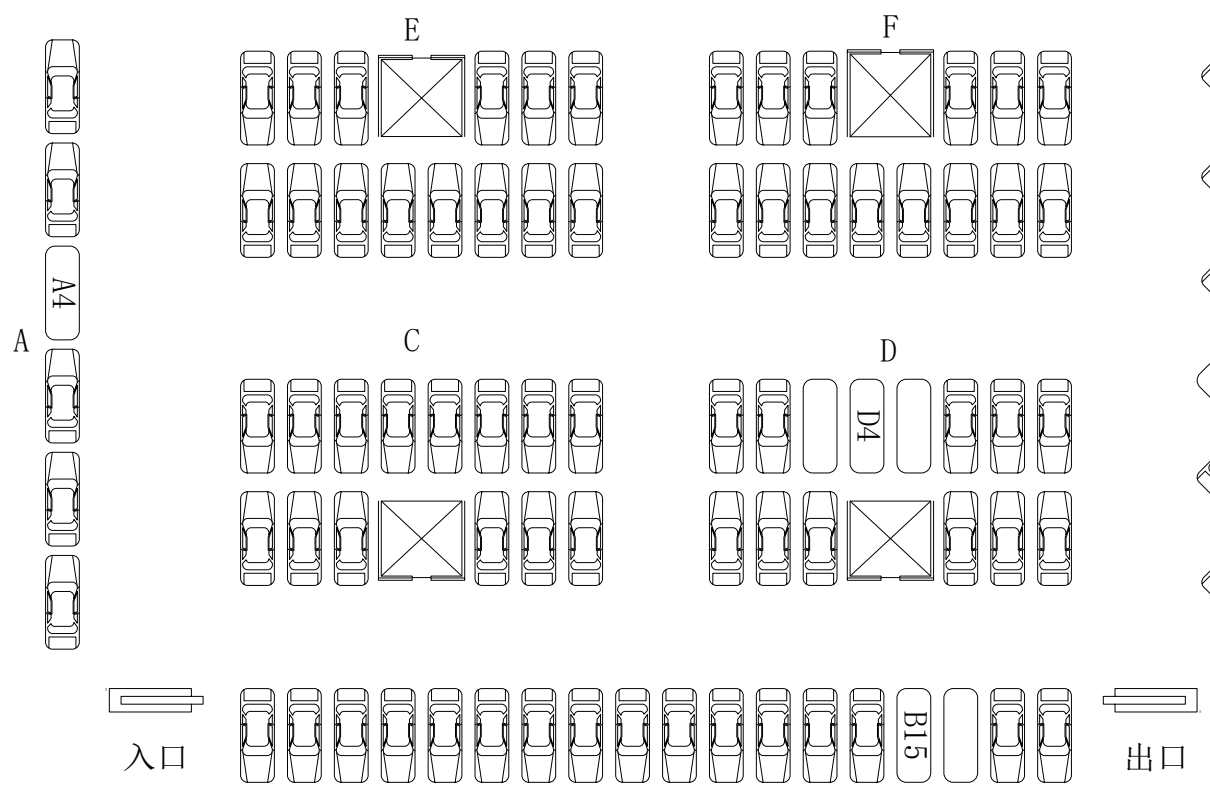

B

Figure 5. The Situations of Free Parking Space

(1) According to 2.1, 2.2 and 2.3, the decision attribute matrix

$$
R^{\prime}=\left[\begin{array}{ccc}
27 & 18 & 0.1 \\
42 & 5 & 0.5 \\
58 & 6 & 0.6 \\
72 & 10 & 0.4 \\
78 & 17 & 0.3
\end{array}\right]
$$

parking spaces A4, B15, D4, G12 and H4 matching the row of $R^{\prime}$;

(2) Getting $R$ by dimensionless standardization;

$$
R=\left[\begin{array}{ccc}
1 & 0 & 0 \\
0.7059 & 1 & 0.8 \\
0.3922 & 0.9231 & 1 \\
0.1176 & 0.6154 & 0.6 \\
0 & 0.0769 & 0.4
\end{array}\right]
$$

(3) The information entropy is $H_{\tilde{i}}=\left[\begin{array}{lllll}0 & 0.4244 & 0.4410 & 0.8570 & 0.5638\end{array}\right]$;

(4) The balanced degree is $B_{i}=\left[\begin{array}{llllll}0 & 0.4952 & 0.5145 & 1 & 0.6579\end{array}\right]$;

(5) The entropy attribute matrix is $V=\left[\begin{array}{ccc}0 & 0 & 0 \\ 0.2996 & 0.4244 & 0.3395 \\ 0.1729 & 0.4071 & 0.4410 \\ 0.1008 & 0.5274 & 0.5142 \\ 0 & 0.0434 & 0.2255\end{array}\right]$;

(6) The ideal solution set is $e^{*}=\left[\begin{array}{lll}0 & 0 & 0.5142\end{array}\right]$;

(7) The grey correlation degree is

$$
R_{\text {oi }}=\left[\begin{array}{lllll}
0.7797 & 0.4843 & 0.5932 & 0.6856 & 0.7787
\end{array}\right]
$$

the equilibrium close degree is 


\section{$\omega=\left[\begin{array}{lllll}0 & 0.2398 & 0.3052 & 0.6856 & 0.5123\end{array}\right]$.}

The maximum value of equilibrium is 0.6856 , its corresponding parking space is G12. It compromise gives an optimal parking space considering driving distance, walking distance and parking space environment information value. Since the shortest path of this parking space has been given by the Dijkstra algorithm, it's the optimal route.

\section{Conclusions}

This paper uses the method of grey entropy relation grade MADM to make decision making about the decision attribute matrix which identified by driving distance, walking distance and parking space environment value, and though the example, it can be proved to realize route optimization to parking lots.

\section{Acknowledgement}

The author would like to thank the anonymous referees for their valuable suggestions. This work was supported by the Key Laboratory Open Fund Project of Intelligent Education and Information Engineering of Heilongjiang Province of China (Grant Nos. SEIE2014-3201423), the Natural Science Foundation of Heilongiiang Province of China (Grant Nos. F201423 and F201426/F030119) and Science and Technology Research Foundation of Heilongjiang Education Department (Grant Nos. 12531162) .

\section{References}

[1] R. W. Wu, "Research on the Key Technologies of smart parking guidance system for large car-park and system development", Nanjing University of Aeronautics and Astronautis Master Dissertation, (2013), p. 1.

[2] L. L. Feng, "Key Technology Research of the Parking Guidance System Based on Internet of Things", Jilin University Master Dissertation, (2013), p. 6.

[3] S. Mukherjee, "Dijkstra's Algorithm fo Solving the Shortest Path Problem on Networks Under Intuitionistic Fuzzy Environment", J Math Model Algor, vol. 10, (2012), pp. 345-347

[4] S. F. Zhang, "Several Methods for Fuzzy Multiple Attribute Decision-making with Applications", Xidian University Doctoral Dissertation, (2012), p. 1.

[5] X. Y. Zhang and K. H. Chen, "The Research of Comprehensive Evaluation System Based on Triangular Fuzzy Number", Sciencepaper Online, (2006), p. 12.

[6] S. Zhang, M. Zhang and G. T. Chi, "The Science and Technology Evaluation Model Based on Entropy Weight and Empirical Research during the 10th Five-Year of China", Chinese Journal of Management, (2010), p. 1.

[7] W. P. Yang and M. L. Lin, "Information Entropy in Intuitionistic Fuzzy Information Systems", Computer Engineering and Applications, vol. 50, no. 11, (2014), pp. 130-134

[8] Q. H. He, W. G. Luo, D. B. Yao, X. P. Cao and J. J. Mao, "A Decision-making Method of Interval Numbers' Grey Situation Based on Grey Entropy”, Journal of Hefei University(Natural Sciences), vol. 2, no. 22, (2012).

[9] Z. Q. Tian, "Research of Transport Structure Based on Information Entropy and Gry Relevance", Inner Mongolia University Master Dissertation, (2013), p. 3.

[10] S. F. Liu, "Emergence and Development of Grey System Theory and Its Forward Trends", Nanjing University of Aeronautics \& Astronautics, (2004), p. 4. 


\section{Authors}

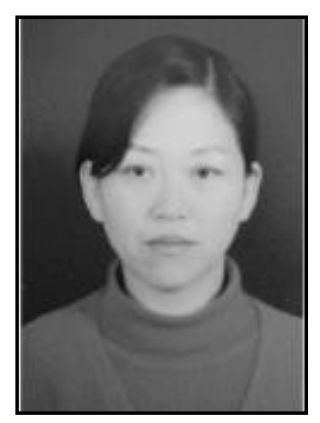

Zhang Yuru is working for Harbin University of Commerce as a professor. Her main research fields include intelligent information processing and fault detection. 